\title{
A Versatile Multiple-Pass Raman System for Industrial Trace Gas Detection
}

\author{
Chunlei Shen, Chengwei Wen, Xin Huang and Xinggui Long *
}

check for updates

Citation: Shen, C.; Wen, C.; Huang, X.; Long, X. A Versatile Multiple-Pass Raman System for Industrial Trace Gas Detection. Sensors 2021, 21, 7173. https://doi.org/10.3390/s21217173

Academic Editor: Anna Chiara De Luca

Received: 28 September 2021

Accepted: 26 October 2021

Published: 28 October 2021

Publisher's Note: MDPI stays neutral with regard to jurisdictional claims in published maps and institutional affiliations.

Copyright: (c) 2021 by the authors. Licensee MDPI, Basel, Switzerland. This article is an open access article distributed under the terms and conditions of the Creative Commons Attribution (CC BY) license (https:// creativecommons.org/licenses/by/ $4.0 /)$.
Institute of Nuclear Physics and Chemistry, China Academy of Engineering Physics, Mianyang 621900, China; shenchunlei@caep.cn (C.S.); chengweiw@caep.cn (C.W.); huangx2016@caep.cn (X.H.)

* Correspondence: xingguil@caep.cn

\begin{abstract}
The fast and in-line multigas detection is critical for a variety of industrial applications. In the present work, we demonstrate the utility of multiple-pass-enhanced Raman spectroscopy as a unique tool for sensitive industrial multigas detection. Instead of using spherical mirrors, D-shaped mirrors are chosen as cavity mirrors in our design, and 26 total passes are achieved in a simple and compact multiple-pass optical system. Due to the large number of passes achieved inside the multiple-pass cavity, experiments with ambient air show that the noise equivalent detection limit $(3 \sigma)$ of 7.6 Pa $\left(\mathrm{N}_{2}\right), 8.4 \mathrm{~Pa}\left(\mathrm{O}_{2}\right)$ and $2.8 \mathrm{~Pa}\left(\mathrm{H}_{2} \mathrm{O}\right)$, which correspond to relative abundance by volume at 1 bar total pressure of $76 \mathrm{ppm}, 84 \mathrm{ppm}$ and $28 \mathrm{ppm}$, can be achieved in one second with a $1.5 \mathrm{~W}$ red laser. Moreover, this multiple-pass Raman system can be easily upgraded to a multiple-channel detection system, and a two-channel detection system is demonstrated and characterized. High utilization ratio of laser energy (defined as the ratio of laser energy at sampling point to the laser output energy) is realized in this design, and high sensitivity is achieved in every sampling position. Compared with single-point sampling system, the back-to-back experiments show that LODs of 8.0 Pa, 8.9 Pa and 3.0 Pa can be achieved for $\mathrm{N}_{2}, \mathrm{O}_{2}$ and $\mathrm{H}_{2} \mathrm{O}$ in one second. Methods to further improve the system performance are also briefly discussed, and the analysis shows that similar or even better sensitivity can be achieved in both sampling positions for practical industrial applications.
\end{abstract}

Keywords: industrial process control; multiple-pass Raman spectroscopy; multiple-point detection; multigas analysis

\section{Introduction}

Optical spectroscopy is one of the most important techniques for multigas analysis since optical spectroscopy techniques are nondestructive and noncontact and allow for in situ monitoring. Traditional multigas analysis techniques include gas chromatography (GC), mass spectroscopy (MS) and infrared (IR) absorption spectroscopy. The analysis speed is relatively slow for GC. Though MS is very sensitive, the instrument is rather expensive, and a lot of calibration efforts are needed for quantitative analysis. Infrared absorption-based technologies, such as tunable diode laser spectroscopy (TDLAS) [1], photoacoustic spectroscopy (PAS) [2] or cavity ring-down spectroscopy (CRDS) [3], are most commonly used since these techniques provide extraordinary sensitivities and selectivity. However, important diatomic homonuclear molecules (e.g., $\mathrm{H}_{2}, \mathrm{~N}_{2}$ ) are challenging to detect with infrared-based techniques. Besides, several laser sources with different wavelengths are required for multigas detection.

Raman spectroscopy, on the other hand, allows for simultaneous identification of almost all gases (e.g., $\mathrm{H}_{2}, \mathrm{CO}_{2}$ and hydrocarbons, except for monatomic gases) with a single laser source. Due to different selection rules, Raman spectroscopy can also be used to target important diatomic homonuclear molecules. These molecules are particularly relevant for many fields, such as power transformer diagnosis [4], medical gas sensing [5,6], biogas analysis $[7,8]$ and process control in nuclear reactors $[9,10]$. The main disadvantage of Raman spectroscopy is the low Raman signal intensity due to small scattering cross section 
of gas molecules and low molecular density in the gas phase. Thus, for Raman spectroscopy to achieve widespread use in scientific and industrial applications, the Raman signal of gas molecules must be enhanced substantially. In the past few years, various Raman systems have been designed and implemented, aiming at lowering limit of detection (LOD) of gas molecules. Examples of such systems are cavity-enhanced Raman spectroscopy (CERS) [11-15], fiber-enhanced Raman spectroscopy (FERS) [16-21], Purcell-enhanced Raman spectroscopy [22,23] and multiple-pass-enhanced Raman spectroscopy [24-31].

Among various techniques, the multiple-pass optical system is the easiest way to realize high sensitivity, though usually the gain factor is limited compared to other techniques. In a multiple-pass system, the optical system design is aimed at increasing the laser energy in a small collection volume, and the multiple reflections of light are ultimately responsible for the resulted high sensitivity. Petrov described a near-concentric multiplepass Raman system based on 90-degree geometry Raman light collection. With $5 \mathrm{~W}$ laser output energy, LODs close to $50 \mathrm{ppm}$ can be achieved in $30 \mathrm{~s}$ for main components of ambient air [26]. Recently, instead of using side detection geometry, Velez et al. employed a collinear detection geometry for their near-concentric multiple-pass cavity, and 34 ppm was achieved for $\mathrm{CO} 2$ in $5 \mathrm{~s}$ [27]. We have recently introduced a variant of multiple-pass Raman spectroscopy with enhanced sensitivity and stability for industrial long-term monitoring applications [29-31]. We take advantage of the large collection area of fiber bundles, which relaxes the laser beam overlap requirements inside a multiple-pass cell. The use of fiber bundle with large area also greatly improves the long-term stability and practicability of an industrial Raman system. With a closed gas chamber, this system is ideal for sensitive in-line monitoring of radioactive or corrosive gas species, as well as other nonhazardous gas samples.

Conventional multiple-pass optical systems for Raman detection usually adopt either (near) concentric or confocal cavity designs. As a result, spherical mirrors are used as cavity mirrors. Usually, the alignment is very tedious in those systems, and cavity mechanical stability is critical. In this contribution, we improve on the multiple-pass optical system developed previously. A highly sensitive and versatile multiple-pass Raman system has been established, mainly aiming for multiple point detection of trace nonhazardous gas samples. Instead of using spherical mirrors, D-shaped flat mirrors are chosen as cavity mirrors in our design, and 26 total passes are achieved inside the compact multiple-pass cavity. Alignment of this multiple-pass system is extremely simple and straightforward. With help of these important improvements, noise equivalent detection limits $(3 \sigma)$ of $7.6 \mathrm{~Pa}$ $\left(\mathrm{N}_{2}\right), 8.4 \mathrm{~Pa}\left(\mathrm{O}_{2}\right)$ and $2.8 \mathrm{~Pa}\left(\mathrm{H}_{2} \mathrm{O}\right)$ are achieved in $1 \mathrm{~s}$ integration time with a $1.5 \mathrm{~W}$ red laser. This multiple-pass Raman system can be easily upgraded to a multiple-channel detection system, and a two-channel detection system is demonstrated and characterized. High utilization ratio of laser energy (defined as the ratio of laser energy at sampling point to the laser output energy) is realized in this design. As a result, high sensitivity is achieved in both sampling positions. Compared with the single-channel system, the back-to-back experiments show that LODs of $8.0 \mathrm{~Pa}, 8.9 \mathrm{~Pa}$ and 3.0 Pa can be achieved for $\mathrm{N}_{2}, \mathrm{O}_{2}$ and $\mathrm{H}_{2} \mathrm{O}$. The results obtained with this multiple-pass Raman setup are very promising, and a variety of industrial applications can benefit from the current design.

\section{Materials and Methods}

The newly designed multiple-pass Raman system is shown schematically in Figure 1. The laser head (Laser Quantum OPUS660) is stabilized by a water cooler, which maintains the base plate temperature at 24 degrees Celsius. The OPUS660, in fact, was first chosen for hydrogen isotopologues monitoring applications in our previous systems [29-31]. We use $660 \mathrm{~nm}$ instead of a shorter wavelength (e.g., $532 \mathrm{~nm}$ ) because, in our previous design, the gas chamber was located between the cavity mirrors, and thus, fluorescence generated from optical windows reduced the signal-to-noise ratio. For current system with a different gas chamber design, 532 $\mathrm{nm}$ or even shorter wavelength can also be used. A band-pass filter (Semrock, FF01-661/11) is used to remove any unwanted laser lines. The laser output beam is then guided by two highly 
reflective mirrors (M1 and M2) to pass an optical isolator. The dielectric coatings of mirror used in this experiment usually have approximately $99.5 \%$ reflectivity at the laser wavelength. After that, a half-wave plate is inserted to tune the polarization of the excitation beam to maximize gas Raman signal for 90-degree collection geometry. The beam is finally focused by a $300 \mathrm{~mm}$ focus lens (L1) into a multiple-pass optical system and reflected multiple times inside the multiple-pass cavity to increase the signal strength.

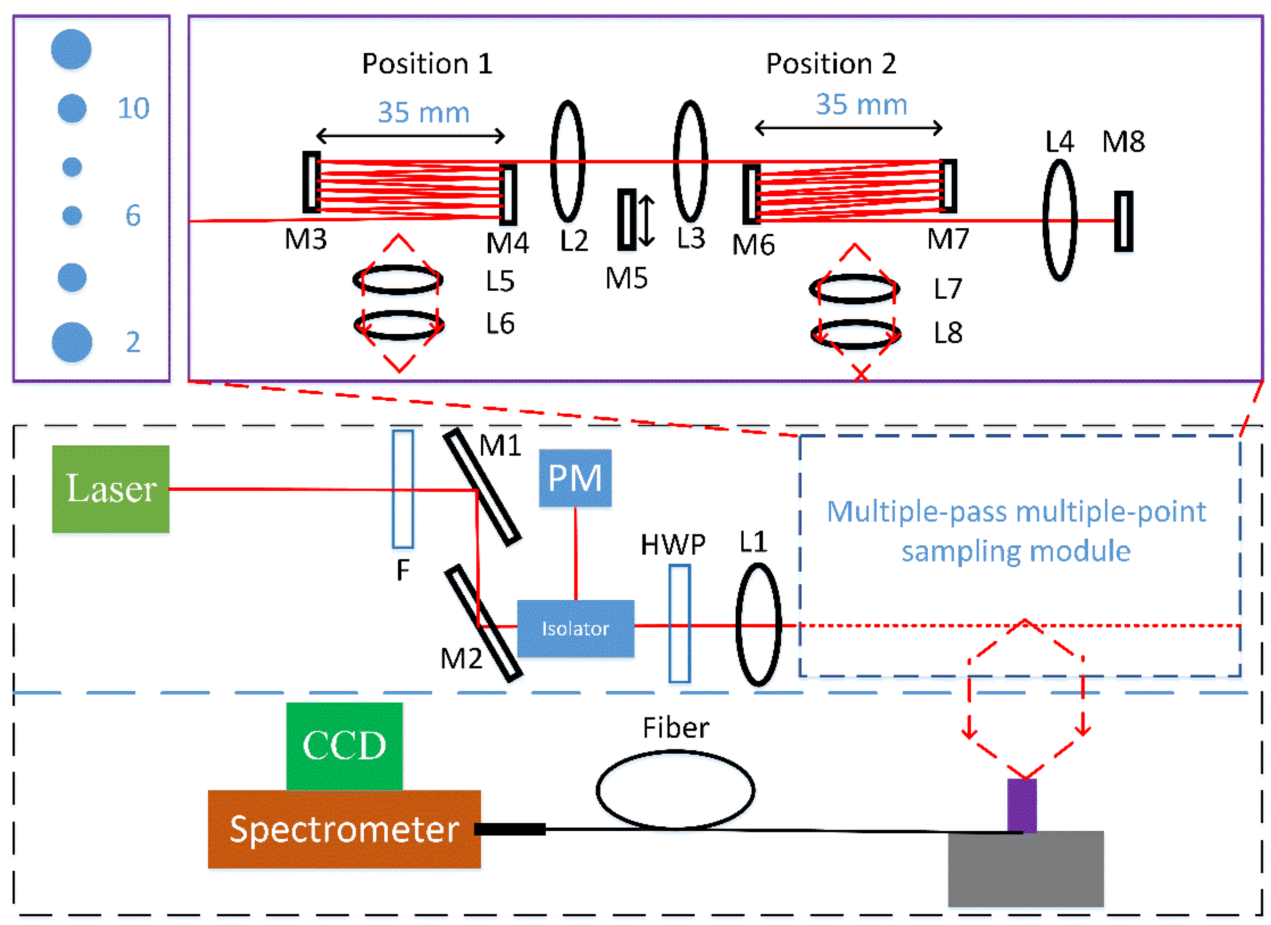

Figure 1. Scheme of the experimental setup. M, Mirrors; L, lenses; F, Filter; PM, power meter; HWP, half-wave plate.

To enhance the Raman signals of nonhazardous gas species in the collection volume, a new multiple-pass scheme is designed. The multiple-pass cell used in our experiments mainly consists of two high-reflection D-shaped mirrors of $25 \mathrm{~mm}$ diameter (M3 and M4), and the alignment of this multiple-pass optical system is greatly simplified by not using spherical mirrors. Those D-shaped mirrors offer an advantage over traditional mirrors since they facilitate the separation of closely spaced beams. The cavity length (distance between M3 and M4) is about $35 \mathrm{~mm}$ and is greatly reduced compared with conventional (near) concentric systems and our previous designs. The distance between M3 and the focusing lens (L1) is approximately $10 \mathrm{~cm}$. The exact distance between optical components is not that important in current design. Alignment of this multiple-pass system is extremely simple, and usually a couple of minutes are enough to complete the construction of the multiple-pass cavity. In the forward path, the incoming beam is first incident on mirror M4. After reflection from this mirror, the beam is incident on the edge of mirror M3. The laser beam is then reflected multiple times between $\mathrm{M} 3$ and $\mathrm{M} 4$ before it leaves the multiple-pass cell defined by M3 and M4. Six laser spots are clearly seen on both mirrors, though the diameters of laser spots are slightly different (spot pattern on M3 is show schematically in Figure 1, top left). The lateral separation of excitation beams in the collection volume is about $8 \mathrm{~mm}$. This excitation geometry gives a total forward pass of 13 (single pass configuration). Using beam diameter of about $1.1 \mathrm{~mm}$ and lens focus of $300 \mathrm{~mm}$, the beam diameter at the focus is $228 \mathrm{um}$ and approximately $700 \mathrm{um}$ for the first and last passes. The beam diameter for other passes will be in between. The out-going beam is then collimated by a second lens with focus of $300 \mathrm{~mm}$ and is finally reflected back by mirror M5 to double the number of passes (double-pass configuration). The back-going beam is finally 
deflected out of the beam path by an isolator to avoid any back-reflection of laser beam into the laser head. Thus, 26 total passes are achieved in this multiple-pass system. During alignment, the laser beams should not clip the sharp edge of the D-shaped mirror in order to minimize formation of interference fringes. Compared with conventional two-concave mirror designs, current multiple-pass system is characterized by its simplicity of alignment and compactness, as well as its adjustment stability.

Another advantage of current design is that the multiple-pass optical system described above can be easily upgraded to a multiple-channel detection system. For example, for a two-channel detection system, the mirror M5 is removed, and lens L3 with a focus of $300 \mathrm{~mm}$ is used to refocus the collimated laser beam into another multiple-pass cell defined by mirrors M6 and M7. The two sampling regions where the Raman signal can be collected are named positions 1 and 2, as also indicated in Figure 1. The incoming beam is then reflected back and forth inside the multiple-pass cavity to give exactly 13 total passes. The out-going laser beam is then collimated by lens L4 with a focus of $300 \mathrm{~mm}$. Finally, mirror M8 is used to double the number of passes in both multiple-pass cavities. Thus, 26 total passes are achieved in both sampling positions.

The gas Raman signals are collected by a pair of achromatic lenses (L5 and L6, with focal lengths of $80 \mathrm{~mm}$ and $50 \mathrm{~mm}$ diameter) at a right angle to the excitation beam and 1:1 imaged onto a fiber bundle comprising 60 multimode fibers (N.A. $=0.22$, core diameter $100 \mathrm{um}$ ) arranged in a rectangular-to-slit configuration. For the two-channel detection system, another pair of achromatic lenses (L7 and L8, with focal lengths of $80 \mathrm{~mm}$ and $50 \mathrm{~mm}$ diameter) is installed to collect the gas Raman signals at position 2. The collection end of the fiber has a dimension of approximately $0.7 \times 1.5 \mathrm{~mm}$ to match the beam diameter in the collection volume. The output end is arranged as a curved slit with approximately $7 \mathrm{~mm}$ height. This allows the full binning of vertical pixels without sacrifice resolution. Typical resolution of our system is about $25 \mathrm{~cm}^{-1}$. For applications where higher resolution is required, either a grating of higher density can be used or multimode fiber with smaller core diameter can be selected. The scattered light is then coupled into a Kaiser Optics $\mathrm{f} / 1.8 \mathrm{i}$ high throughout spectrograph. This system contains no moving parts to ensure long system stability and is suitable for industrial applications. The Raman spectra were finally recorded by a CCD detector (PIXIS 400BRX) operating at -74 degrees Celsius.

\section{Results}

\subsection{Performance of Current Multiple-Pass Raman System}

Compared with our previous multiple-pass setups, the multiple-pass cavity length is greatly reduced in the new design, and it is impossible to insert a closed gas chamber between cavity mirrors. The current setup can be directly used to monitor gas species in an atmosphere environment. For example, multiple consecutive breaths from different people can be exhaled into the sampling positions using Teflon tubes [24,25]. For applications where a closed gas chamber is needed, a slight modification of current configuration can be adopted, and the multiple-pass cavities (M3, M4 and M6, M7) can be placed inside two closed gas chambers $[4,11,26]$. For example, the system can be applied to power transformer diagnosis and logging gas detection, and the gas samples can be sent to the (multiple) closed gas chambers through a valve system. Both configurations have the advantage that no fluorescence background is generated in the excitation region. To demonstrate performance and sensitivity of this multiple-pass Raman system, spectra of ambient air were recorded without a gas cell.

For the double pass configuration, the spectrum of ambient air is shown in Figures 2 and 3. For these experiments, the laser output power was set to $1.5 \mathrm{~W}$. The spectrum of ambient air is dominated by the spectral features of oxygen $\left(\mathrm{O}_{2}\right)$ at $735.5 \mathrm{~nm}\left(1555.2 \mathrm{~cm}^{-1}\right)$, nitrogen $\left(\mathrm{N}_{2}\right)$ at $779.9 \mathrm{~nm}\left(2329.9 \mathrm{~cm}^{-1}\right)$ and water molecules $\left(\mathrm{H}_{2} \mathrm{O}\right)$ at $869.9 \mathrm{~nm}\left(3656.2 \mathrm{~cm}^{-1}\right)$. Besides major components, the spectral features of $\mathrm{Q}_{2}\left(\mathrm{~N}_{2}\right)$ branch at $952.8 \mathrm{~nm}\left(4656.0 \mathrm{~cm}^{-1}\right)$ and $\mathrm{CO}_{2}$ at $721.1 \mathrm{~nm}\left(1283.6 \mathrm{~cm}^{-1}\right)$ and $726.6 \mathrm{~nm}\left(1388.7 \mathrm{~cm}^{-1}\right)$ can also be unequivocally assigned with $1 \mathrm{~s}$ integration time (Figure 2, bottom), though the contrast of the higher 
wavenumber peak of the $\mathrm{CO}_{2}$ Fermi resonance pair has been degraded by the $\mathrm{Q}_{1}\left(\mathrm{O}_{2}\right)$ branch. The signature of $\mathrm{Q}_{2}\left(\mathrm{O}_{2}\right)$ branch at $828.9 \mathrm{~nm}\left(3087.6 \mathrm{~cm}^{-1}\right)$ is almost noise-limited with $1 \mathrm{~s}$ integration time but is detectable with $10 \mathrm{~s}$ integration time, as shown in Figure 3. The above results clearly demonstrate the high sensitivity achieved in this Raman setup, considering that the intensity of $\mathrm{Q}_{2}\left(\mathrm{O}_{2}\right)$ branch is approximately 3000 times less than $\mathrm{Q}_{1}$ $\left(\mathrm{O}_{2}\right)$ branch. The LODs are estimated using procedures described in detail in a previous publication and is associated with a signal-to-noise ratio (SNR) of 3 [29]. In the current work, noise is calculated using flat regions between 5400 to $5700 \mathrm{~cm}^{-1}$. For $\mathrm{N}_{2}$ and $\mathrm{O}_{2}$ (assuming $78 \%$ and $21 \%$ composition in the air) with $1 \mathrm{~s}$ integration time, the noise $(\sigma)$ is extracted as 1.96. The estimated noise equivalent detection limits $(3 \sigma)$ are $7.6 \mathrm{~Pa}$ and 8.4 Pa for $\mathrm{N}_{2}$ and $\mathrm{O}_{2}$, respectively, which corresponds to relative abundance by volume at 1 bar total pressure of $76 \mathrm{ppm}$ and $84 \mathrm{ppm}$. From the humidity data logger reading of $55 \% \mathrm{RH}$ (corresponding to approximately $1265 \mathrm{~Pa}$ of water vapor in the air), a LOD of 2.8 $\mathrm{Pa}$ is deduced for $\mathrm{H}_{2} \mathrm{O}$, which corresponds to relative abundance by volume at 1 bar total pressure of $28 \mathrm{ppm}$. Recently, Godot et al. have introduced a commercial Raman analyzer for process control in a tritium facility, and the LOD was reported as $200 \mathrm{~Pa}$ for hydrogen isotopologues, with an acquisition time of $120 \mathrm{~s}$ [32]. Based on our previous investigations [29-31], the LOD of the current setup for hydrogen isotopologues can be safely estimated to be lower than that of $\mathrm{H}_{2} \mathrm{O}$. Thus, the current system is preferred in low-pressure gas applications. In recent year, FERS has shown excellent sensitivity for multigas analysis. Hanf et al. showed that LODs of $9 \mathrm{ppm}$ and $8 \mathrm{ppm}$ could be achieved for $\mathrm{N}_{2}$ and $\mathrm{O}_{2}$ [33]. For $\mathrm{H}_{2} \mathrm{~S}$, LOD as low as 33 ppm was also demonstrated [20]. Besides, small samples volumes are needed in this technique, which is critical for certain applications where amount of gas sample is limited. However, the small core diameter hampers the quick exchange of gas samples, and this technique is not suitable for in-line low-pressure gas detection and monitoring.

We have also tested a slightly different version of the single-pass configuration, where the isolator and half-wave plate are removed. A power meter is also inserted between L3 and L2 to safely collect the laser light and monitor laser performance. First, this configuration gives a simpler Raman system and should improve system robustness, as the number of optical components is reduced. Besides, full laser energy can be used for excitation, as approximately $15 \%$ laser energy is lost after passing the isolator and half-wave plate. The recorded spectra of ambient air are also shown in Figures 2 and 3 with different integration times. Compared with double-pass configuration, the LODs for gas molecules have been increased by a factor of about 1.6. For certain applications, the achieved sensitivity is still acceptable, and single-pass configuration provides a simpler and lower-cost solution.

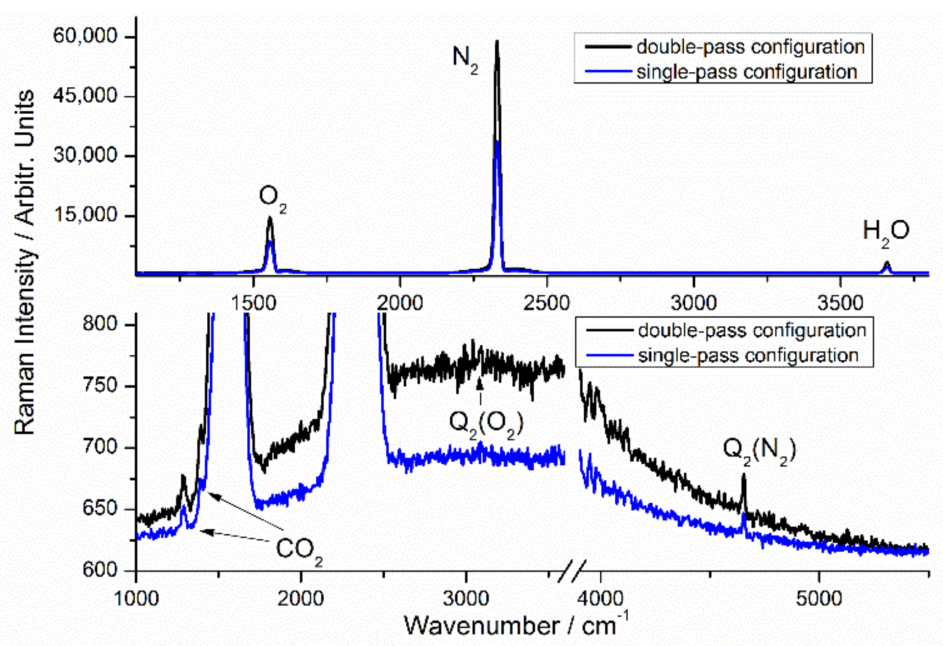

Figure 2. Raw spectra of ambient air with $1 \mathrm{~s}$ integration time. Top: Spectral overview. Bottom: Low-intensity parts of spectra. 


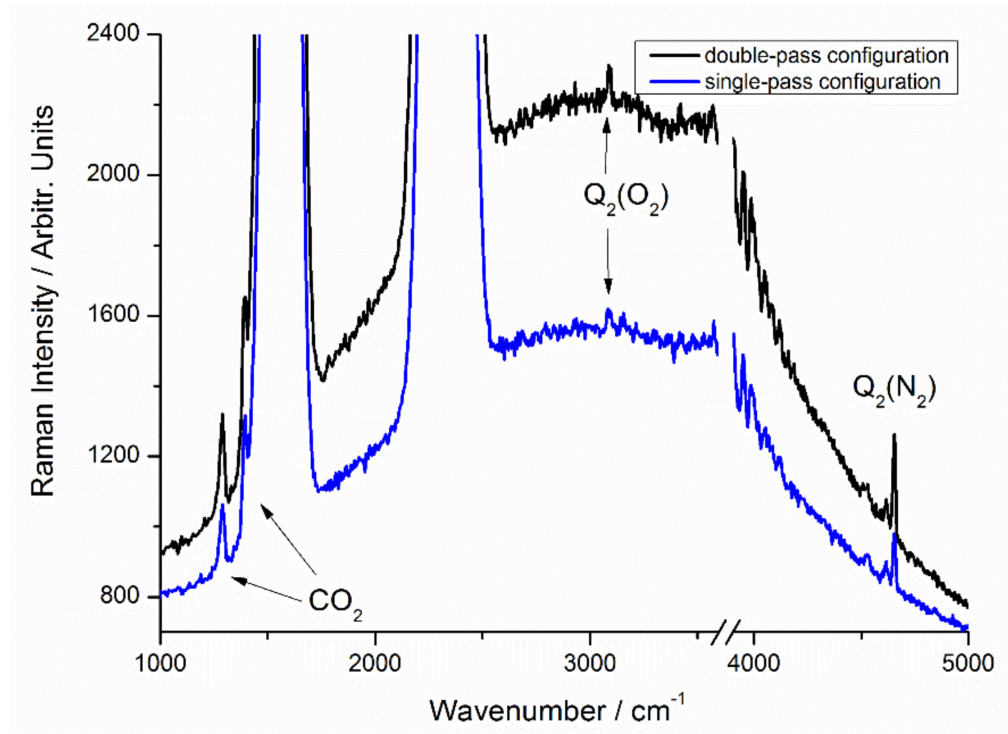

Figure 3. Low-intensity parts of raw spectra with $10 \mathrm{~s}$ integration time. Note that with $10 \mathrm{~s}$ integration time, the Q-branch peaks (not shown) of $\mathrm{O}_{2}$ and $\mathrm{N}_{2}$ are saturated in the detector.

\subsection{Characterization of the Two-Channel Detection System}

With the development of science and technology, industrial monitoring applications also have even higher requirements for gas sensor systems. Besides high sensitivity and long-term stability, some applications require that the Raman system can be operated in an economical manner. The multiple-channel detection scheme greatly reduces the examination costs of a monitoring system and thus has drawn extensive attention in industrial multigas analysis applications. In real industrial gas detection applications, different gas samples can be transported to different detection positions (e.g., different gas chambers) through valve-pipeline systems. Thus, simultaneous composition monitoring at different sampling positions are realized using the same laser source and spectrometer. To demonstrate the sensitivity of this newly designed two-channel detection system, spectra of ambient air were recorded back-to-back at positions 1 and 2. The detailed experimental procedure is as follows: The spectra of lab air were recorded first in position 1. After data collection in position 1, the fiber bundle was removed and reinstalled and optimized in position 2. The spectra of lab air were then recorded in position 2. It should be noted that for these experiments the same fiber bundle is used, though in practical situations, signals can be collected simultaneously at multiple sampling positions through a branched fiber bundle.

For the two-channel detection system, the spectra of ambient air recorded with laser output set to be $1.5 \mathrm{~W}$ is shown in Figure 4. The spectra of ambient air (Figure 4, top) recorded in positions 1 and 2 are nearly indistinguishable by visual inspection. The small difference in signal strength is due to slightly different alignments. With $10 \mathrm{~s}$ integration time, the peaks of $\mathrm{Q}_{2}\left(\mathrm{~N}_{2}\right)$ and $\mathrm{CO}_{2}$ are readily identified, and the peak of $\mathrm{Q}_{2}\left(\mathrm{O}_{2}\right)$ is also distinguishable (Figure 4, bottom). Thus, similar high-sensitivity is also achieved in a two-channel detection system. At position 1 with $1 \mathrm{~s}$ integration time, experiments with ambient air show that the noise equivalent detection limit $(3 \sigma)$ of $8.0 \mathrm{~Pa}\left(\mathrm{~N}_{2}\right)$, 8.9 $\mathrm{Pa}\left(\mathrm{O}_{2}\right)$ and $3.0 \mathrm{~Pa}\left(\mathrm{H}_{2} \mathrm{O}\right)$ can be achieved, which corresponds to relative abundance by volume at $1 \mathrm{bar}$ total pressure of $80 \mathrm{ppm}, 89 \mathrm{ppm}$ and $30 \mathrm{ppm}$. The LODs calculated at position 2 are almost identical to values obtained with position 1 . The estimated LODs are slightly higher than the above (double-pass configuration) single-channel detection system, which is reasonable since the laser energy loss is higher in a two-channel detection system. 


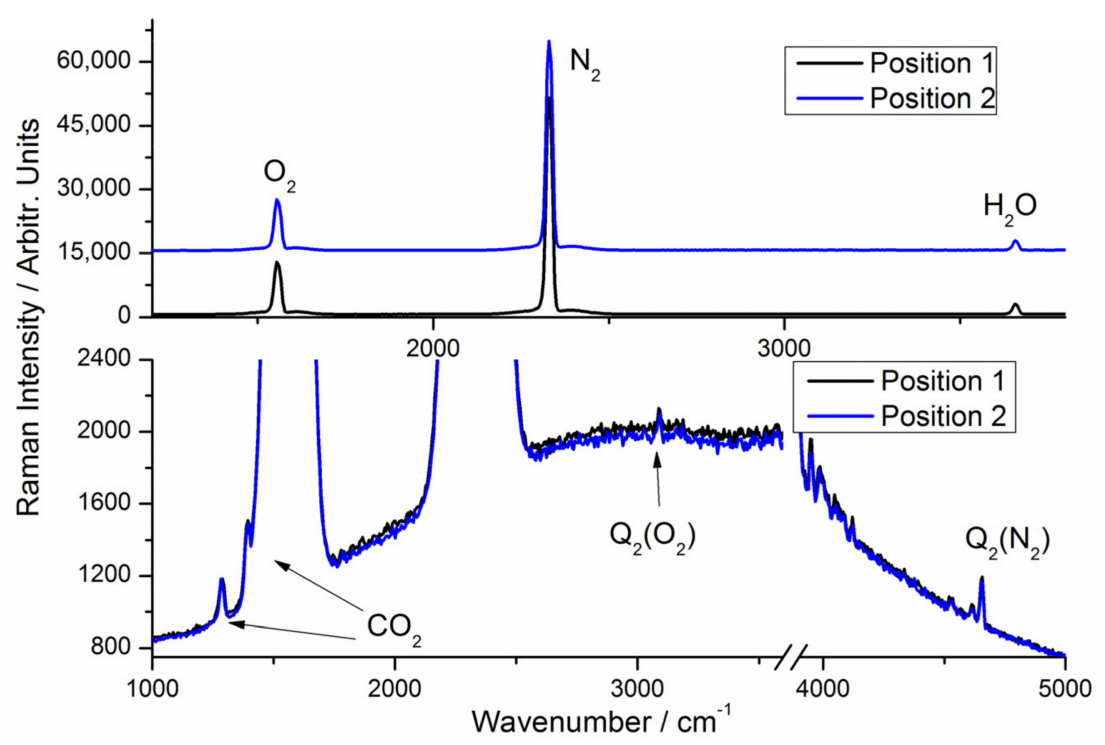

Figure 4. Raw spectra of ambient air at sampling positions 1 and 2. Top: Spectral overview with $1 \mathrm{~s}$ integration time. Traces are offset by 15,000 units. Bottom: Low-intensity parts of spectra with $10 \mathrm{~s}$ integration.

The above results clearly demonstrate sensitivity and capability of this Raman setup for multigas analysis. Due to similar design criteria, the long-term stability should also be comparable to our previous setups. Thus, this newly designed Raman system is especially suited for trace gas analysis in a number of industrial applications. Besides, the signal enhancement provided with current multiple-pass system can be further improved. Various approaches to improve the system performance have already been discussed thoroughly in a previous contribution, and we are not going to discuss them in detail [29,31]. For example, back reflection mirrors can be installed to double the collection solid angle; the total number of achievable passes can also be increased (e.g., 30 total passes) by adjusting the relative position (angle and distance) of the two cavity mirrors.

\section{Conclusions}

Sensitive multigas composition analysis and monitoring are needed for a variety of industrial applications. Usually, a gas analyzer that measures gas samples in a wide range of concentration and in real-time is required for process control applications. To this end, a highly sensitive, versatile and economical multiple-pass Raman system has been established and characterized, mainly aiming for multiple-point detection of trace nonhazardous gas samples. Instead of using spherical mirrors, D-shaped mirrors are chosen as cavity mirrors in this design, and 26 total passes are achieved inside a compact multiple-pass cavity. With $1.5 \mathrm{~W}$ of $660 \mathrm{~nm}$ excitation laser and $1 \mathrm{~s}$ integration time, cavity enhancement achieves LODs of 7.6 Pa $\left(\mathrm{N}_{2}\right), 8.4 \mathrm{~Pa}\left(\mathrm{O}_{2}\right)$ and $2.8 \mathrm{~Pa}\left(\mathrm{H}_{2} \mathrm{O}\right)$.

Moreover, this multiple-pass Raman system can be easily upgraded to a multiplechannel detection system, and a two-channel detection system is demonstrated and characterized. Compared with single-channel detection system, the noise equivalent detection limits at the two positions are almost identical and have been estimated to be $8.0 \mathrm{~Pa}\left(\mathrm{~N}_{2}\right)$, 8.9 $\mathrm{Pa}\left(\mathrm{O}_{2}\right)$ and 3.0 $\mathrm{Pa}\left(\mathrm{H}_{2} \mathrm{O}\right)$. The slightly higher LODs obtained in the two-channel detection system are mainly due to reflection loss at the mirrors and lenses, and the achieved LODs can be improved by using customized coatings. Other methods to improve the system performance are also briefly discussed, and the analysis indicates that similar or even better sensitivity can be achieved for every sampling position in a practical twochannel detection system. The results obtained with this multiple-pass Raman setup are very promising. The sensitivity can be further improved by using lasers with higher power since the LODs almost scale linearly with excitation laser power. Additionally, the Raman 
signal can be significantly increased by using lasers with shorter wavelengths. Thus, limits of detection in low ppm range for common gas samples are possible with exposure times of seconds.

Author Contributions: Conceptualization, C.W. and X.L.; investigation, C.S. and C.W.; writingoriginal draft preparation, C.S. and X.H.; supervision, X.L.; All authors have read and agreed to the published version of the manuscript.

Funding: This research received no external funding.

Institutional Review Board Statement: Not applicable.

Informed Consent Statement: Not applicable.

Data Availability Statement: The data that support the findings of this study are available from the corresponding author upon reasonable request.

Conflicts of Interest: The authors declare no conflict of interest.

\section{References}

1. Reid, J.; Labrie, D. Second-Harmonic Detection with Tunable Diode Lasers? Comparison of Experiment and Theory. Appl. Phys. A 1981, 26, 203-210. [CrossRef]

2. Li, S.; Dong, L.; Wu, H.; Sampaolo, A.; Patimisco, P.; Spagnolo, V.; Tittel, F.K. Ppb-Level Quartz-Enhanced Photoacoustic Detection of Carbon Monoxide Exploiting a Surface Grooved Tuning Fork. Anal. Chem. 2019, 91, 5834-5840. [CrossRef]

3. Banik, G.D.; Som, S.; Maity, A.; Pal, M.; Maithani, S.; Mandal, S.; Pradhan, M. An EC-QCL based N2O Sensor at $5.2 \mu \mathrm{m}$ using Cavity Ring-Down Spectroscopy for Environmental Applications. Anal. Methods 2017, 9, 2315-2320. [CrossRef]

4. Wang, P.; Chen, W.; Wang, J.; Tang, J.; Shi, Y.; Wan, F. Multigas Analysis by Cavity-Enhanced Raman Spectroscopy for Power Transformer Diagnosis. Anal. Chem. 2020, 92, 5969-5977. [CrossRef]

5. Chow, K.K.; Short, M.; Lam, S.; McWilliams, A.; Zeng, H. A Raman Cell Based on Hollow Core Photonic Crystal Fiber for Human Breath Analysis. Med. Phys. 2014, 41, 092701. [CrossRef] [PubMed]

6. Hanf, S.; Bögözi, T.; Keiner, R.; Frosch, T.; Popp, J. Fast and Highly Sensitive Fiber-Enhanced Raman Spectroscopic Monitoring of Molecular H2 and CH4 for Point-of-Care Diagnosis of Malabsorption Disorders in Exhaled Human Breath. Anal. Chem. 2014, 87, 982-988. [CrossRef]

7. Kiefer, J.; Seeger, T.; Steuer, S.; Schorsch, S.; Weikl, M.; Leipertz, A. Design and Characterization of a Raman-Scattering-Based Sensor System for Temporally Resolved Gas Analysis and its Application in a Gas Turbine Power Plant. Meas. Sci. Technol. 2008, 19, 085408. [CrossRef]

8. Knebl, A.; Domes, R.; Wolf, S.; Domes, C.; Popp, J.; Frosch, T. Fiber-Enhanced Raman Gas Spectroscopy for the Study of Microbial Methanogenesis. Anal. Chem. 2020, 92, 12564-12571. [CrossRef] [PubMed]

9. O'Hira, S.; Hayashi, T.; Nakamura, H.; Kobayashi, K.; Todokoro, T.; Nakamura, H.; Itoh, T.; Yamanishi, T.; Kawamura, Y.; Iwai, Y.; et al. Improvement of Tritium Accountancy Technology for ITER Fuel Cycle Safety Enhancement. Nucl. Fusion. 2000, 40, 519-526. [CrossRef]

10. Demange, D.; Alecu, C.G.; Bekris, N.; Borisevich, O.; Bornschein, B.; Fischer, S.; Gramlich, N.; Köllö, Z.; Le, T.L.; Michling, R.; et al. Overview of R\&D at TLK for Process and Analytical Issues on Tritium Management in Breeder Blankets of ITER and DEMO. Fusion Eng. Des. 2012, 87, 1206-1213.

11. Salter, R.; Chu, J.; Hippler, M. Cavity-Enhanced Raman Spectroscopy with Optical Feedback Cw Diode Lasers for Gas Phase Analysis and Spectroscopy. Analyst 2012, 137, 4669-4676. [CrossRef] [PubMed]

12. Hippler, M. Cavity-Enhanced Raman Spectroscopy of Natural Gas with Optical Feedback cw-Diode Lasers. Anal. Chem. 2015, 87, 7803-7809. [CrossRef] [PubMed]

13. Taylor, D.J.; Glugla, M.; Penzhorn, R.-D. Enhanced Raman Sensitivity Using an Actively Stabilized External Resonator. Rev. Sci. Instrum. 2001, 72, 1970-1976. [CrossRef]

14. Sieburg, A.; Schneider, S.; Yan, D.; Popp, J.; Frosch, T. Monitoring of Gas Composition in a Laboratory Biogas Plant Using Cavity Enhanced Raman Spectroscopy. Analyst 2018, 143, 1358-1366. [CrossRef]

15. Frosch, T.; Keiner, R.; Michalzik, B.; Fischer, B.; Popp, J. Investigation of Gas Exchange Processes in Peat Bog Ecosystems by Means of Innovative Raman Gas Spectroscopy. Anal. Chem. 2013, 85, 1295-1299. [CrossRef]

16. Pearman, W.F.; Carter, J.C.; Angel, S.M.; Chan, J.W.-J. Multipass Capillary Cell for Enhanced Raman Measurements of Gases. Appl. Spectrosc. 2008, 62, 285-289. [CrossRef] [PubMed]

17. Buric, M.P.; Chen, K.P.; Falk, J.; Woodruff, S.D. Multimode Metal-Lined Capillaries for Raman Collection and Sensing. J. Opt. Soc. Am. B 2010, 27, 2612-2619. [CrossRef]

18. Okita, Y.; Katagiri, T.; Matsuura, Y. A Raman Cell Based on Hollow Optical Fibers for Breath Analysis. Proc. SPIE 2010, 7559, 755908. [CrossRef] 
19. James, T.M.; Rupp, S.; Telle, H.H. Trace Gas and Dynamic Process Monitoring by Raman Spectroscopy in Metal-Coated Hollow Glass Fibres. Anal. Methods 2015, 7, 2568-2576. [CrossRef]

20. Sieburg, A.; Knebl, A.; Jacob, J.M.; Frosch, T. Characterization of Fuel Gases with Fiber-Enhanced Raman Spectroscopy. Anal. Bioanal. Chem. 2019, 411, 7399-7408. [CrossRef] [PubMed]

21. Wolf, S.; Frosch, T.; Popp, J.; Pletz, M.W.; Frosch, T. Highly Sensitive Detection of the Antibiotic Ciprofloxacin by Means of Fiber Enhanced Raman Spectroscopy. Molecules 2019, 24, 4512. [CrossRef]

22. Petrak, B.; Djeu, N.; Muller, A. Purcell-Enhanced Raman Scattering from Atmospheric Gases in a High-Finesse Microcavity. Phys. Rev. A 2014, 89, 023811. [CrossRef]

23. Petrak, B.; Cooper, J.; Konthasinghe, K.; Peiris, M.; Djeu, N.; Hopkins, A.J.; Muller, A. Isotopic Gas Analysis through Purcell Cavity Enhanced Raman Scattering. Appl. Phys. Lett. 2016, 108, 091107. [CrossRef]

24. Borysow, J.; Fink, M. NIR Raman Spectrometer for Monitoring Protonation Reactions in Gaseous Hydrogen. J. Nucl. Mater. 2005, 341, 224-230. [CrossRef]

25. Schlüter, S.; Krischke, F.; Popovska-Leipertz, N.; Seeger, T.; Breuer, G.; Jeleazcov, C.; Schüttler, J.; Leipertz, A. Demonstration of a Signal Enhanced Fast Raman Sensor for Multi-Species Gas Analyses at a Low Pressure Range for Anesthesia Monitoring. J. Raman Spectrosc. 2015, 46, 708-715. [CrossRef]

26. Petrov, D.V. Multipass Optical System for a Raman Gas Spectrometer. Appl. Opt. 2016, 55, 9521-9525. [CrossRef] [PubMed]

27. Velez, J.G.; Muller, A. Trace Gas Sensing Using Diode-Pumped Collinearly Detected Spontaneous Raman Scattering Enhanced by a Multipass Cell. Opt. Lett. 2020, 45, 133-136. [CrossRef]

28. Li, X.; Xia, Y.; Zhan, L.; Huang, J. Near-Confocal Cavity-Enhanced Raman Spectroscopy for Multitrace-Gas Detection. Opt. Lett. 2008, 33, 2143-2145. [CrossRef]

29. Wen, C.; Huang, X.; Shen, C. Multiple-Pass Enhanced Raman Spectroscopy for Fast Industrial Trace Gas Detection and Process Control. J. Raman Spectrosc. 2020, 51, 781-787. [CrossRef]

30. Wen, C.; Huang, X.; Shen, C. Multiple-Pass Enhanced Multiple-Point Gas Raman Analyzer for Industrial Process Control Applications. J. Raman Spectrosc. 2020, 51, 2046. [CrossRef]

31. Wen, C.; Huang, X.; Wang, W.; Shen, C.; Li, H. Multiple-Pass Enhanced Raman Spectroscopy for Long-Term Monitoring of Hydrogen Isotoplogues. J. Raman Spectrosc. 2019, 50, 1555. [CrossRef]

32. Godot, A.; Coindet, G.; Hubinois, J.C. Analysis of Gases by Raman Spectroscopy: Determination of Isotopic Composition of Hydrogen Mixtures (H2, D2 and T2). Fusion Sci. Technol. 2011, 60, 998-1001. [CrossRef]

33. Hanf, S.; Keiner, R.; Yan, D.; Popp, J.; Frosch, T. Fiber-Enhanced Raman Multigas Spectroscopy: A Versatile Tool for Environmental Gas Sensing and Breath Analysis. Anal. Chem. 2014, 86, 5278-5285. [CrossRef] [PubMed] 